\title{
A study of the mercury levels in Scottish dentists
}

Mercury vapour levels in dental practices and body mercury levels of dentists and controls

\author{
K. A. Ritchie, F. J. T. Burke, W. H. Gilmour, E. B. Macdonald, I. M. Dale, R. M. Hamilton, D. A. McGowan, \\ V. Binnie, D. Collington and R. Hammersley Br Dent J 2004; 197: 625-632
}

\begin{abstract}
Aim
A study of 180 dentists in the West of Scotland was conducted to determine their exposure to mercury during the course of their work and the effects on their health and cognitive function.

Design

Data were obtained from questionnaires distributed to dentists and by visiting their surgeries to take measurements of environmental mercury.

\section{Methods}

Dentists were asked to complete a questionnaire including items on handling of amalgam, symptoms experienced, diet and possible influences on psychomotor function such as levels of stress and alcohol intake. They also completed the 12-item General Health Questionnaire. Dentists were asked to complete a dental chart of their own mouths and to give samples of urine, hair and nails for mercury analysis. The dentists were visited at their surgeries where environmental measurements were made in eight areas of the surgery and they undertook a computerised package of psychomotor tests. One hundred and eighty control subjects underwent a similar procedure, completing a questionnaire, having their amalgam surfaces counted, giving urine, hair and nail samples and undergoing the psychomotor test procedure.
\end{abstract}

\section{Results}

Dentists were found to have, on average, urinary mercury levels over 4 times that of control subjects although all but one dentist had urinary mercury below the Health and Safety Executive health guidance value of $20 \mu \mathrm{mol} \mathrm{mmol}{ }^{-1}$ creatinine. Urine was found to be a better biological marker for mercury exposure than hair or nails. Dentists were significantly more likely than control subjects to have suffered from disorders of the kidney but these symptoms were not significantly associated with their level of mercury exposure as measured in urine. One hundred and twenty two (67.8\%) of the 180 surgeries visited had environmental mercury measurements in one or more areas above the Occupational Exposure Standard (OES) set by the Health and Safety Executive. In the majority of these surgeries the high levels of mercury were found at the skirting and around the base of the dental chair. In 45 surgeries (25\%) the personal dosimetry measurement (ie in the breathing zone of dental staff) was above the OES.

\section{Conclusion}

On the basis of these findings, it is recommended that greater emphasis should be made relating to safe handling of amalgam in the training and continuing professional development of dentists, that further studies are carried out on levels of mercury exposure of dental team members during the course of their working day, and that periodic health surveillance, including urinary mercury monitoring, of dental personnel should be conducted to identify possible effects of practising dentistry.

\section{COMMENT}

Psychologists, dental academics, NHS advisers, occupational health scientists, public health commentators; all were involved in assembling the data presented in this paper and they subjected it to a rigorous analysis. An old adage says that laying such experts end to end will never produce an agreement; well in this case there was a consensus view.

The experts had extensively questioned 180 Scottish dentists in an attempt to find out whether placing amalgams was affecting their health. Samples from each dentist had been taken away for analysis and a team of scientists had checked their surgeries with the intent of discovering whether hazardous sources of mercury were lurking in dark corners. Finally the dentists had been subjected to psychomotor tests, the results of which are to be published on another occasion.

Not surprisingly, when the bits of nail, hair and urine were analysed and compared with similar samples taken from those not working with amalgam, the body burden of mercury was found to be four times higher in those using amalgam. However, except for one user, the mercury in their urine was found to be below that suggested as a safe level by the Health and Safety Executive (HSE).

When the surgeries were examined, two thirds had levels of mercury vapour above the occupational safety standard set by the HSE, suggesting that more emphasis should be given to good mercury hygiene practices in the teaching and guidance of all members of the dental team, and that tests should be carried out both on personnel and the environment to identify 'the possible effects of practising dentistry' - now there's a topic for Panorama.

D. Brown, Senior Lecturer, Dental Biomaterials Science, GKT Dental Institute doi:10.1038/sj.bdj.4811829

\section{IN BRIEF}

- One hundred and eighty dental surgeries were tested for environmental mercury.

- Sixty eight per cent had environmental mercury readings over the occupational exposure standard.

- Greater emphasis is needed in the safe handling of mercury.

- Dentists were more likely to have suffered a kidney disorder than the control group. 